\title{
BMJ open Cost-effectiveness and quality of life in surgeon versus general practitioner-organised colon cancer surveillance: a randomised controlled trial
}

\author{
Knut Magne Augestad, ${ }^{1,2,3}$ Jan Norum, ${ }^{3,4}$ Stefan Dehof, ${ }^{5}$ Ranveig Aspevik, ${ }^{5}$ \\ Unni Ringberg, ${ }^{6}$ Torunn Nestvold, ${ }^{7}$ Barthold Vonen, ${ }^{3,7}$ Stein Olav Skrøvseth, ${ }^{1}$ \\ Rolv-Ole Lindsetmo ${ }^{2,3}$
}

To cite: Augestad KM, Norum J, Dehof S, et al. Cost-effectiveness and quality of life in surgeon versus general practitioner-organised colon cancer surveillance: a randomised controlled trial. BMJ Open 2013:3:e002391. doi:10.1136/bmjopen-2012002391

- Prepublication history for this paper are available online. To view these files please visit the journal online (http://dx.doi.org/10.1136/ bmjopen-2012-002391).

Received 22 November 2012 Revised 28 January 2013 Accepted 14 February 2013

This final article is available for use under the terms of the Creative Commons Attribution Non-Commercial 2.0 Licence; see http://bmjopen.bmj.com

For numbered affiliations see end of article.

Correspondence to Dr Knut Magne Augestad; knut.magne.augestad@ telemed.no

\section{ABSTRACT}

Objective: To assess whether colon cancer follow-up can be organised by general practitioners (GPs) without a decline in the patient's quality of life (QoL) and increase in cost or time to cancer diagnoses, compared to hospital follow-up.

Design: Randomised controlled trial.

Setting: Northern Norway Health Authority Trust, 4 trusts, 11 hospitals and 88 local communities. Participants: Patients surgically treated for colon cancer, hospital surgeons and community GPs. Intervention: 24-month follow-up according to national guidelines at the community GP office. To ensure a high follow-up guideline adherence, a decision support tool for patients and GPs were used. Main outcome measures: Primary outcomes were QoL, measured by the global health scales of the European Organisation for Research and Treatment of Cancer QoL Questionnaire (EORTC QLQ C-30) and EuroQol-5D (EQ-5D). Secondary outcomes were costeffectiveness and time to cancer diagnoses.

Results: 110 patients were randomised to intervention $(n=55)$ or control $(n=55)$, and followed by $78 \mathrm{GPs}$ (942 follow-up months) and 70 surgeons (942 followup months), respectively. Compared to baseline, there was a significant improvement in postoperative QoL $(p=0.003)$, but no differences between groups were revealed (mean difference at 1, 3, 6, 9, 12, 15, 18, 21 and 24-month follow-up appointments): Global Health; $\Delta-2.23, p=0.20 ; E Q-5 D$ index; $\Delta-0.10, p=0.48, E Q-5 D$ VAS; $\Delta-1.1, p=0.44$. There were no differences in time to recurrent cancer diagnosis (GP 35 days vs surgeon 45 days, $p=0.46$ ); 14 recurrences were detected (GP 6 vs surgeon 8 ) and 7 metastases surgeries performed (GP 3 vs surgeon 4). The follow-up programme initiated 1186 healthcare contacts (GP 678 vs surgeon 508 ), 1105 diagnostic tests (GP 592 vs surgeon 513 ) and 778 hospital travels (GP 250 vs surgeon 528 ). GP organised follow-up was associated with societal cost savings (£8233 vs $£ 9889, p<0.001)$.

Conclusions: GP-organised follow-up was associated with no decline in QoL, no increase in time to recurrent cancer diagnosis and cost savings.

\section{ARTICLE SUMMARY}

Article focus

- Intensive follow-up after curative colon cancer resection is associated with improved overall survival of $5-10 \%$.

- No international consensus exists regarding the detailed content of a follow-up programme for colorectal cancer.

- Quality of life (QoL), cost-effectiveness and patient safety in a general practitioner (GP)-organised follow-up programme are unknown.

\section{Key messages}

- GP-organised colon cancer follow-up is associated with no decline in QoL, no increase in time to recurrent cancer diagnosis and cost savings.

Strengths and limitations of this study

- Intention to treat analyses with high adherence to the national follow-up programme.

- First trial assessing cost-effectiveness of a GP-organised colon cancer follow-up programme.

- The trial was stopped after 1884 patient follow-up months owing to no impact of the intervention on QoL global health status.

- Fifty two per cent of the included patients were followed for 2 years. This limits the interpretation of recurrence, as $80 \%$ of the colon cancer recurrences occur within 3 years.

Trial registration: ClinicalTrials.gov identifier NCT00572143.

\section{BACKGROUND}

Colon cancer is the third most common cancer in the western world, and surgery is the only curative treatment. Around one-third of the patients resected of colon cancer will 
experience recurrence of the disease with less than 2 years expected survival. ${ }^{12}$ Despite the generally poor outcomes among patients with recurrent disease, most patients treated with curative intent are included in some form of surveillance programme involving periodic evaluation. Reviews comparing various follow-up programmes have suggested that more intensive strategies tend to increase 5 -year survival by detecting relapse about 6 months earlier than less intensive strategies-at a point where the patient will be more likely to be considered a candidate for potentially curative metastases surgery. ${ }^{2-4}$ However, wide consensus has not been reached regarding what an intensive follow-up strategy should entail. ${ }^{5-8}$ New surveillance trials in progress are not likely to fully settle the issue.$^{9-12}$ What none of the available clinical recommendations for follow-up have addressed adequately is the setting where this follow-up should occur: conducted by specialists who originally treated the cancer at hospitals, or in the offices of local GPs. ${ }^{2}$ Increasingly, the benefits of greater involvement of primary care providers in the ongoing management of chronic illnesses are recognised. ${ }^{83}$ The level of follow-up care may greatly influence the quality of life (QoL) and costs, especially in rural areas with long distances to travel for hospital services. However, such considerations must be balanced against the imperative that colon cancer survivors receive the best care available. Recently, the UK's National Cancer Survivorship Initiative recognised the need to develop new models of cancer care that support patient self care, care planning and making the best out of resources. ${ }^{14}$ In Norway, similar national initiatives have been launched. In this trial, we tested the main hypothesis that patients with colon cancer followed up by their GP would experience similar or higher scores on QoL measures at a lower cost than alternative hospital controls. The other aims were to test for differences of harms and benefits in a follow-up programme, that is, the rate of serious clinical events (SCE), time to diagnosis of SCE and cancer recurrence and frequency of metastases surgery.

\section{METHODS}

This was a randomised controlled multicentre trial carried out in a North Norway Health Authority trust using a previously published protocol. ${ }^{15}$ The first patient was included on 1 June 2007, while the last patient was included on 15 December 2011. Interim analyses were performed in June 2012.

\section{Ethics and trial registration}

The Regional Committee for Medical Research Ethics, North Norway approved this protocol in 2006 (P REK NORD 79/2006). Patients provided written consent before entering the trial. The trial was registered at ClinicalTrials.gov with identifier NCT00572143. Owing to organisational delay, the trial was registered on 11 December 2007; the specified study start in ClinicalTrials.gov was June 2007.
Inclusion and exclusion criteria

Inclusion criteria were age less than 75 years with recent surgery for colon cancer at Dukes' stage A, B or C. Patients receiving postsurgical adjuvant chemotherapy (some Dukes' B and all Dukes' C) were also eligible. Exclusion criteria were patients older than 75 years, patients belonging to healthcare trust not participating in the trial or those not able to provide informed consent and cancer stage Dukes' D.

\section{Hospitals, primary and secondary care professionals}

Three local hospitals and one university hospital participated. Approximately 100 patients with colon cancer are surgically treated annually at these four hospitals. All 550 GPs in the region received written information, while 448 GPs consented to participate in the trial.

\section{Objective and hypotheses}

The primary objective was to compare patients' QoL and costs of follow-up by their local GP or at the surgical outpatient clinic. The primary hypothesis was that the patients followed up by their GPs would experience similar or better QoL scores (on the global health scale) at a lower cost. The secondary objective was to test whether the incidence of SCE would be similar for patients followed up by their GPs or hospital surgeons the secondary hypothesis being that patients followed up by their GPs would have no delay in detection of relapse and the same frequency of SCEs as controls.

\section{Description of intervention}

We defined this as a complex intervention, consisting of several interconnecting parts. ${ }^{16}$ To ensure high follow-up guideline adherence by patients allocated to GPs' followups, we used a decision support tool as part of the intervention. ${ }^{17}$ Thus, the intervention consisted of the following parts:

1. GP organised colon cancer follow up: The patients were referred to their GP for postoperative follow-up according to the national guidelines (table 1). Information was given to the GP about surgery, any complications, Dukes' staging, time and location of chemotherapy (for Dukes' C patients), and risk of recurrence.

2. Patient decision-support pamphlet: Received at the baseline consultation, containing information about: (1) their own disease, tumour stage and risk of recurrence; (2) the aim and objective of the trial; (3) the current national follow-up guidelines, that is, schedule and location of carcinoembryonic antigen (CEA) measurements, chest x-ray, contrast-enhanced liver ultrasound, colonoscopy and clinical examination; (4) a detailed description of signs and symptoms of potential recurrence of colon cancer and (5) in case of a SCE between appointments, relevant phone numbers and contact information were given.

3. GP decision-support pamphlet: Sent at the time of baseline appointment to all GPs who had a patient allocated to their practice. This pamphlet contained 
Table 1 Norwegian Gastrointestinal Cancer Group (NGICG) 2007 surveillance programme

\begin{tabular}{|c|c|c|c|c|c|c|c|c|c|c|c|c|c|c|c|}
\hline \multirow[b]{2}{*}{ Examination } & \multicolumn{15}{|c|}{ Follow-up cycle (month postoperative) } \\
\hline & 1 & 3 & 6 & 9 & 12 & 15 & 18 & 21 & 24 & 30 & 36 & 42 & 48 & 54 & 60 \\
\hline Chest x-ray & & & $\mathrm{X}$ & & $\mathrm{X}$ & & $\mathrm{X}$ & & $\mathrm{X}$ & & $x$ & & $\mathrm{X}$ & & $\mathrm{X}$ \\
\hline Liver ultrasonography & & & $\mathrm{X}$ & & $\mathrm{x}$ & & $\mathrm{x}$ & & $\mathrm{X}$ & & $X$ & & $\mathrm{X}$ & & $\mathrm{X}$ \\
\hline Colonoscopy & & & & & $\mathrm{X}$ & & & & & & & & $\mathrm{X}$ & & \\
\hline CEA measurement & $\mathrm{X}$ & $\mathrm{X}$ & $\mathrm{X}$ & $\mathrm{X}$ & $\mathrm{X}$ & $\mathrm{X}$ & $\mathrm{X}$ & $\mathrm{X}$ & $X$ & $X$ & $x$ & $x$ & $x$ & $\mathrm{X}$ & $x$ \\
\hline Clinical examination & $x$ & $\mathrm{X}$ & $x$ & $x$ & $\mathrm{x}$ & $x$ & $\mathrm{X}$ & $\mathrm{X}$ & $\mathrm{X}$ & $\mathrm{X}$ & $\mathrm{X}$ & $x$ & $x$ & $\mathrm{X}$ & $x$ \\
\hline
\end{tabular}

information similar to what the patient received, that is, information about follow-up guidelines, signs and symptoms of recurrence and behavioural strategy in the case of suspicion of a recurrence. In case of questions regarding the follow-up, relevant contact information was given.

Patients allocated to GP follow-up could be referred back to any surgical clinic at any time during the study period. Similarly, patients in the hospital follow-up group were free to consult their GP at any time. National follow-up guidelines were applied in both study arms and patients were followed for up to 2 years (table 1).

\section{Randomisation}

At study entry, patients were seen for a baseline visit by a local trial investigator at the hospital where they received the surgical treatment, approximately 3-4 weeks postoperatively. At this visit, a clinical examination was performed and information about the histology and results of the surgery was shared with each patient. If the patients provided informed consent, they were randomised to follow-up either by their GP (intervention) or at the surgical outpatient clinic (controls) using a webbased randomisation service managed by the Norwegian University of Science and Technology (http://www.ntnu. no). The randomisation ratio was $1: 1$; patients were stratified according to the Dukes' staging (A, B and C) and on whether they had a stoma. The local trial investigator was not involved in the subsequent follow-up appointments in any way. Recruited patients were not informed about the other patients recruited in the same trial. Similarly, no information regarding trial progress and allocation was revealed to the participating GPs or surgeons. However, as GP organised follow-up represented a new practice, blinding was not possible in the intervention arm.

\section{Primary outcome measures}

Quality of life

The primary outcome measure in this trial was the global health status on the European Organisation for Research and Treatment of Cancer QoL Questionnaire (EORTC QLQ C-30). QoL measurements were collected at baseline and at 3, 6, 9, 12, 15, 18, 21 and 24 months, that is,

European Organisation for Research and Treatment of Cancer QoL Questionnaire: EORTC QLQ C-30 incorporates nine multi-item scales: five functional scales (physical, role, cognitive, emotional and social); three symptom scales (fatigue, pain and nausea/vomiting) and a global health status/QOL scale. Six single-item scales are also included (dyspnoea, insomnia, appetite loss, constipation, diarrhoea and financial difficulties). ${ }^{18}$

EuroQol-5D (EQ-5D; EuroQol Group, Rotterdam, The Netherlands): EuroQol-5D is a standardised generic QoL instrument. EQ-5D measures five dimensions of health-related QoL (HRQoL): mobility, self care, usual activities, pain/discomfort and anxiety/depression. Each dimension is rated at three levels: no problems (1), some problems (2) and major problems (3). ${ }^{19}$ Based on preferences elicited from a general population, EQ-5D health states (eg, 1-1-2-1-3) may be converted into utility scores (=index scores, IS). In this trial, we used preferences elicited from a UK population, as no similar Norwegian preferences exist. ${ }^{20}$

EQ visual analogue scale (EQ VAS) records the respondent's self-rated health status on a vertically graduated (0-100) visual analogue scale.

\section{Secondary outcome measures \\ Cost-effectiveness}

Resources used (baseline to 24 months) were registered prospectively based on reports by the patients and on hospital electronic medical record (EMR) review. The cost elements included costs related to hospital visits, GP visits, laboratory tests, radiology examinations, colonoscopy, examinations owing to suspected relapse (radiology, colonoscopy, CT of thorax and/or abdomen and positron emission tomography scan), treatment of recurrence, travelling/transportation, production losses, copayments and other patient/family expenses.

\section{Time to cancer diagnosis}

Time to cancer diagnosis was defined as the time from occurrence of an SCE (dated in the GP referral or hospital EMR record) to the date of diagnosis of an SCE. An SCE was defined as an episode in which cancer recurrence was suspected. An SCE can be triggered either by symptoms reported (at follow-up or in between 
follow-ups), clinical findings at follow-up or findings by a screening test. Symptoms and clinical findings initiating a diagnostic check-up were defined as follows: cancer suspect lesion revealed at colonoscopy, increase in CEA measurements shown by repeated measurements, blood in stool detected by the Hemofec (FOB) test, unexplained abdominal pain, unexplained weight loss of $5 \mathrm{~kg}$ during the last 3 months, cancer-suspect lesions detected by rectal examination, palpable lymphadenopathy, metastatic suspect lesions shown by chest x-ray, ultrasound of liver or CT scan, cancer-suspect findings at clinical examination and occurrence of cancer-related symptoms.

\section{Data collection}

At the baseline appointment, patients recruited received nine questionnaires (as part of the patient decisionsupport pamphlet) corresponding with the nine follow-up cycles (table 1). The questionnaires contained questions about QoL, patient satisfaction and cost and resource utilisation. Questionnaires were returned by mail every 3 months by the patients to the trial centre until 24 months postoperatively. These questionnaires were optically readable, being consecutively registered in the trial database. A research assistant was responsible for data collection, database input and patient reminders when questionnaires were missing. The reminders were sent to the participating patients when the questionnaires were 3 months overdue (beyond the estimated follow-up schedule). All questionnaires were dated and we could thus monitor trial progression. In case of missing information about cost elements, we either reviewed the hospital EMR or performed telephone interviews with participating surgeons, GPs or patients.

\section{Sample size calculation}

In June 2007, sample size calculations were based on a significance level of $5 \%$ and power set at $80 \%$, which indicated that we needed 136 patients to detect a 10 units QoL difference (ie, a small to moderate improvement) on the EORTC QLQ C-30 Global Health score with an SD of 20. Definitions of a small-tomoderate improvement on QoL (ie, 10 units on the global health score), and SD estimates of QoL (patients with localised colon cancer) were retrieved from previous published publications. ${ }^{21} 22$

\section{Economic analysis}

$B M J$ guidelines for economic analyses alongside randomised controlled trials were employed. ${ }^{23}$ As the trial revealed no difference in QoL, a cost-minimisation analysis was carried out. The economic evaluation had a societal perspective. A 3\% discount rate was used to discount future costs and benefits. For this publication, cost elements have been converted from Norwegian kroner (NOK) into British Pounds at the rate of GBP $£ 1=\mathrm{NOK}$ 9.39 NOK as of the Norwegian National Bank on 27 June 2012. Details of the unit costs assigned to healthcare resource use are shown in table 2.
Economic evaluation data are invariably positively skewed, and they require an alternative analysis. We used a bootstrapping technique, which makes no assumptions regarding the equality, variance or shape of the distribution, and takes skewness into account. ${ }^{24} 25$ To adjust for the skewness, costs were bootstrapped with 1000 replications to estimate bias corrected CI. The bootstrapping technique was undertaken using IBM SPSS Statistics V.19.0.

A one-way sensitivity analysis was used to assess the robustness of the results and the impact of variance. The societal cost of 24-month follow-up was assessed for low, base and high-input values, and the result was expressed as a many inputs, one output tornado chart. To increase the generalisability of cost between countries, unit costs from the UK were included in the sensitivity analyses. The costs for GP consultation and diagnostic testing have been reported to be $30-40 \%$ higher than the unit cost applied in this trial and the relevant cost elements were increased accordingly in sensitivity analyses. ${ }^{26}$

\section{Statistics}

Descriptive statistics were performed by percentages, $2 \times 2$ contingency tables, $\chi^{2}$, Fisher's exact test and t test. The base case analyses $(n=110,600$ complete follow-up questionnaires/cycles) were performed on the intention to treat principle. Treatment arms were compared with respect to potential covariates using continuous and categorical univariable analyses. The main analyses examined whether significant differences existed in QoL outcome measures between baseline and 3, 6, 9, 12, 15, 18, 21 and 24 months (EORTC QLQ C-30 and EQ-5D). A general linear model was employed, where time (1-24 months) and intervention group (GPs vs surgeons) were predictors in analyses of variance (between groups ANOVA). Missing data were imputed by the last observation carried forward (LOCF) when there were missing QoL items in a form, and when the QoL form was missing. Conditional power (CP) was defined as the chance of getting statistically significant results at the end of the trial given the data so far. ${ }^{27}$ We defined a $\mathrm{CP}<15 \%$ as a sufficient threshold to stop early. ${ }^{29}$ Results were expressed as the mean differences for continuous outcomes with corresponding SD, 95\% CIs, and associated $p$ values. $p$ Values were reported with two decimal places with $p$-values less than 0.001 being reported as $\mathrm{p}<0.001$. For all tests, we used the $\mathrm{p}=0.05$ level of significance. All analyses were performed with the IBM SPSS Statistics V.19.0 (IBM Company SPSS 2010) and Microsoft Excel for Mac 2011.

\section{RESULTS}

A total of 110 patients surgically treated for colon cancer met the inclusion criteria and agreed to participate in the survey (figure 1). The control and intervention groups were matched at baseline for demographic and 
Table 2 Details of the unit costs assigned to healthcare resource use data

\begin{tabular}{|c|c|c|}
\hline Variable & Unit cost $(£)^{\star}$ & Sensitivity analyses (\%) \\
\hline Cost of travel & & \pm 25 \\
\hline Mean costs of hospital travel & $88 \dagger$ & \\
\hline Hotel overnight & $74 \ddagger$ & \\
\hline Private car rates & $0.2 / \mathrm{km} \S$ & \\
\hline Parking & $10.6 \ddagger$ & \\
\hline Taxi & $1.3 / \mathrm{km} \S$ & \\
\hline Bus & $2.6 \S$ & \\
\hline Cost of GP consultation & & $\pm 25-40$ \\
\hline GP consultation $20 \mathrm{~min}$ & $18.5 \rrbracket$ & \\
\hline Phone consultation GP $10 \mathrm{~min}$ & 5.39 & \\
\hline Emergency consultation GP $30 \mathrm{~min}$ & $26 \rrbracket$ & \\
\hline Cost of surgeon outpatient consultation & & $\pm 25-40$ \\
\hline Surgeon outpatient consultation $30 \mathrm{~min}$ & $69^{\star \star}$ & \\
\hline Phone consultation surgeon $15 \mathrm{~min}$ & $10.6 \dagger †$ & \\
\hline Emergency outpatient consultation 30 min & $69^{\star \star}$ & \\
\hline Cost of follow-up tests & & $\pm 25-40$ \\
\hline Blood samples & 59 & \\
\hline Chest x-ray & $25 \ddagger \ddagger, \S \S$ & \\
\hline Contrast-enhanced ultrasound of liver & $153 \ddagger \ddagger, \S \S$ & \\
\hline CT abdomen & $105 \ddagger \ddagger, \S \S$ & \\
\hline CT thorax & $105 \ddagger \ddagger, \S \S$ & \\
\hline Colonoscopy & $293^{\star \star}, \S \S$ & \\
\hline PET scan & $2662 \ddagger \ddagger$ & \\
\hline Cost related to sick leave & & \pm 25 \\
\hline Governmental reimbursement 1 day work absence & 102ףП & \\
\hline Costs related to metastases surgery & & \pm 25 \\
\hline Cost of abdominal surgery & $14176^{\star \star}$ & \\
\hline Cost of liver surgery & $11596^{\star \star}$ & \\
\hline Cost of lung surgery & $13061^{\star *}$ & \\
\hline \multicolumn{3}{|c|}{ 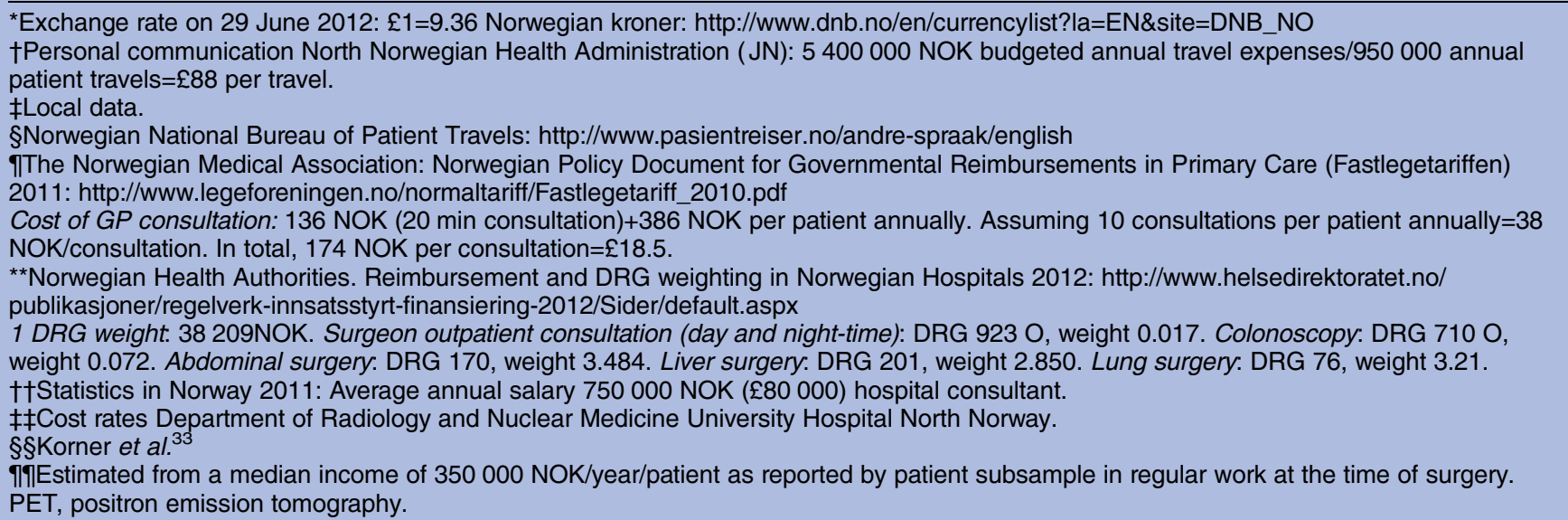 } \\
\hline
\end{tabular}

medical characteristics and there were no significant differences between groups (table 3).

\section{Trial flow and dropouts}

Eighty five patients (75\%) (GP 41 vs surgeon 44) were followed for 12 months, and 58 patients (52\%) (GP 29 vs surgeon 29) for 24 months. Twenty patients (surgeon 9 vs GP 11) were transferred to the new national colon cancer surveillance programme (figure 1).

\section{Response rate}

We received 636 of the expected 657 questionnaires (response rate 96\%), of which 600 (91\%) (GP 299 vs surgeon 301) were included in the final cost and QoL analyses. A total of $21(4 \%)$ questionnaires (surgeon 11 vs GP 10) were not returned and 36 questionnaires (surgeon 18 vs GP 18) were excluded from the analyses owing to insufficient identification.

\section{Interim analyses}

New national colon cancer surveillance guidelines were gradually implemented from 2010, with a different frequency of consultations (3 vs 6 months interval) and radiological modalities (chest x-ray vs chest CT). ${ }^{7}$ This could bias the cost-effectiveness and QoL 


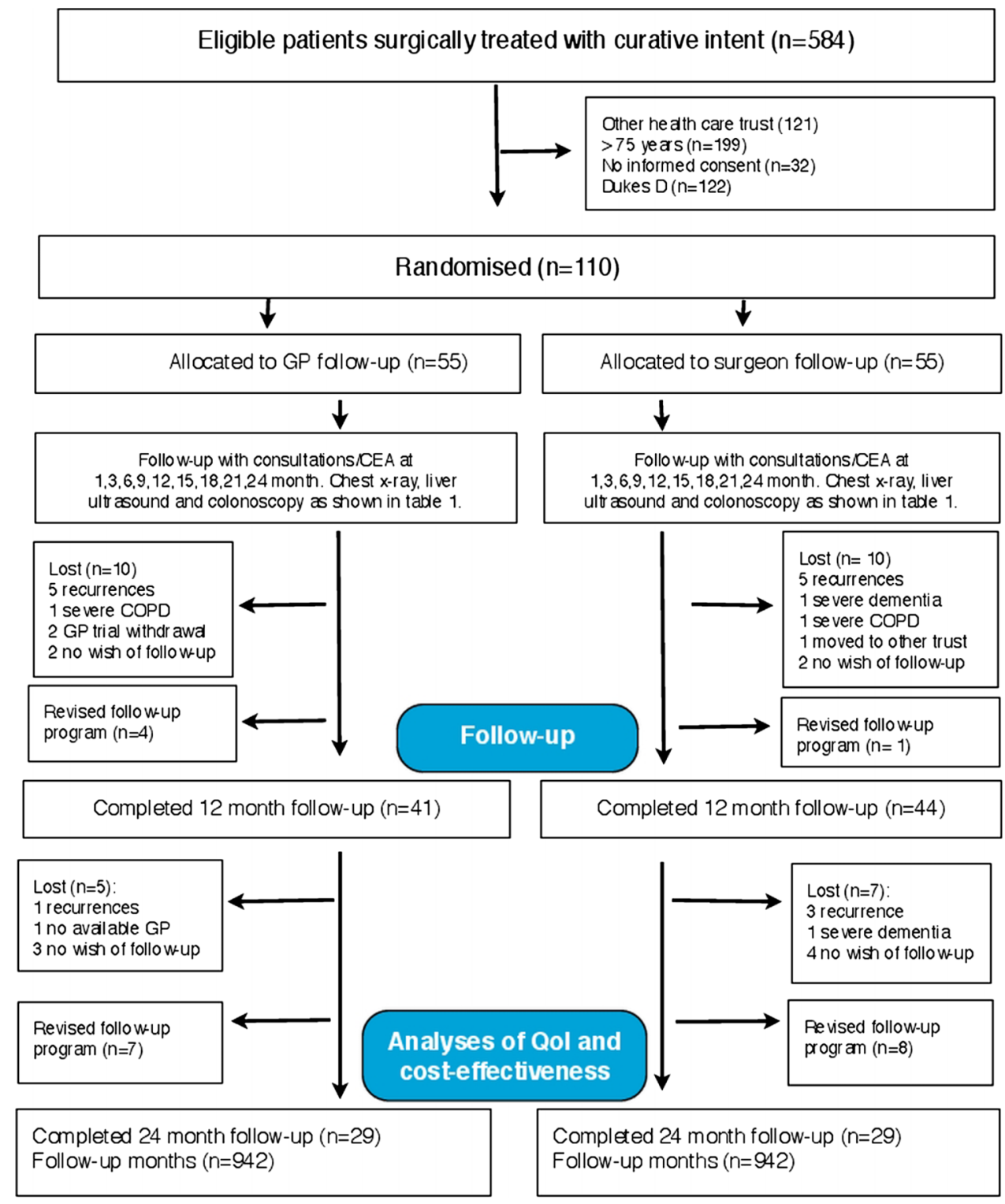

Figure 1 Flow of participants. Patients were enrolled in the 2007 NGICG (Norwegian Gastrointestinal Cancer Group, table 1) follow-up programmes in both trial arms. The programmes are divided in 3-month cycles, that is, clinical examination at 1 (baseline), 3, 6, 9, 12, 15, 18, 21 and 24 months, carcinoembryonic antigen (CEA) measurement at 3-month intervals, chest x-ray and contrast-enhanced liver ultrasound every 6 months and colonoscopy once during 24 months (table 1).

analyses, and an interim analysis was performed in June 2012 (80\% of preplanned recruitment, 1884 follow-up months). There was at this point a $4 \%$ probability (ie, conditional power) of showing a significant impact of the intervention on QoL global health score, which meant that further trial continuation were not justified.

\section{Quality of life}

There was no significant effect on the QoL main outcome measures. However, on the EORTC QLQ C-30 subscales, there were significant effects in favour of GP follow-up, that is, role functioning $(p=0.02)$, emotional functioning $(\mathrm{p}=0.01)$ and pain $(\mathrm{p}=0.01$; table 4 and figure $2 \mathrm{~A}-\mathrm{C})$.

\section{Cost-effectiveness}

There were no significant differences in primary QoL measures (global health status), and cost minimisation analyses were performed. A total of 778 travels (consultations, radiological investigations and colonoscopy) to the hospital were registered, 528 in the surgeon group and 250 in the GP group, respectively. A total of 1186 healthcare contacts (regular appointments, emergency appointments and phone consultations) were registered, 678 in the GP group versus 508 in the surgeon group (table 5). The mean cost of follow-up per patient per follow-up cycle was £292 in the GP group and £351 in the surgeon group $(p=0.02$ ) (figure 3 ). Overall, the mean societal cost per patient for 24 months follow-up was $£ 9889$ in the surgeon group and $£ 8233$ in the GP group $(\mathrm{p}<0.001$, table 6$)$. 
Table 3 Baseline demographics and clinical characteristics

\begin{tabular}{|c|c|c|c|c|}
\hline Variable & Surgeon $(\%) n=55$ & GP (\%) $n=55$ & Total $(\%) n=110$ & p Value \\
\hline \multicolumn{5}{|l|}{ Age group } \\
\hline$<50$ & $2(3.6)$ & $6(10.9)$ & 7 (6.3) & 0.10 \\
\hline $50-59$ & $8(14.5)$ & 6 (10.9) & $14(12.7)$ & 0.56 \\
\hline $60-69$ & $23(41.8)$ & $24(43.6)$ & $47(42.7)$ & 0.84 \\
\hline $70-75^{\star}$ & $22(40.0)$ & 19 (34.5) & $41(38.0)$ & 0.55 \\
\hline Mean age (SD) & $66.7(7.3)$ & $64.0(8.7)$ & $65.4(8.1)$ & 0.09 \\
\hline \multicolumn{5}{|l|}{ Gender } \\
\hline Male & 32 (58.2) & $33(60.0)$ & $65(59.1)$ & 0.84 \\
\hline Female & $23(41.8)$ & $22(40.0)$ & 45 (40.9) & 0.84 \\
\hline \multicolumn{5}{|l|}{ Education } \\
\hline Primary & $20(36.3)$ & $18(32.7)$ & $38(34.5)$ & 0.68 \\
\hline Secondary & $21(38.1)$ & $25(45.4)$ & $46(41.8)$ & 0.49 \\
\hline University $<4$ years & $8(14.5)$ & $5(9.0)$ & $13(11.8)$ & 0.37 \\
\hline University $>4$ years & $6(10.9)$ & $7(12.7)$ & $13(11.8)$ & 0.76 \\
\hline \multicolumn{5}{|l|}{ Income level } \\
\hline Median (£) & $32-42000$ & $32-42000$ & $32-42000$ & \\
\hline \multicolumn{5}{|l|}{ Main activity } \\
\hline Employment & $12(21.8)$ & 17 (30.9) & $29(26.3)$ & 0.27 \\
\hline Home & $3(5.4)$ & 9 (16.3) & $11(10.0)$ & 0.06 \\
\hline Out of work & $0(0)$ & $1(1.8)$ & $1(0.9)$ & \\
\hline Pensioner & $40(72.7)$ & $28(50.9)$ & $68(61.8)$ & 0.01 \\
\hline \multicolumn{5}{|l|}{ Location of surgery } \\
\hline University hospital $(n=1)$ & $34(61.8)$ & $37(67.3)$ & $71(64.5)$ & 0.55 \\
\hline Local hospital $(n=3)$ & $21(38.1)$ & $18(32.7)$ & 39 (35.4) & 0.55 \\
\hline \multicolumn{5}{|l|}{ Clinical characteristics } \\
\hline \multicolumn{5}{|l|}{ Tumour location } \\
\hline Cøkum & $13(23.6)$ & $13(23.6)$ & $26(23.6)$ & 1.0 \\
\hline Ascendens & $9(16.3)$ & $5(9.1)$ & $14(12.7)$ & 0.25 \\
\hline Transversum & $4(7.2)$ & $5(9.1)$ & $9(8.1)$ & 0.72 \\
\hline Decendens & $1(1.8)$ & $4(1.8)$ & $5(4.5)$ & 0.15 \\
\hline Sigmoid & $28(50.9)$ & $28(50.9)$ & $56(50.9)$ & 1.0 \\
\hline Elevated preoperative CEA & $19(34.5)$ & $23(41.8)$ & $42(38.1)$ & 0.55 \\
\hline \multicolumn{5}{|l|}{ Type of surgery } \\
\hline Laparoscopic surgery & $14(25.5)$ & $11(20.0)$ & $25(22.7)$ & 0.49 \\
\hline Open surgery & $41(74.5)$ & $44(80.0)$ & 85 (77.3) & 0.49 \\
\hline \multicolumn{5}{|l|}{ Tumour stage } \\
\hline Dukes A & $12(21.8)$ & $11(20.0)$ & $24(21.8)$ & 0.81 \\
\hline Dukes B & $25(45.5)$ & $30(54.5)$ & $55(50.0)$ & 0.34 \\
\hline Dukes C & $18(32.7)$ & $14(25.5)$ & $32(29.0)$ & 0.40 \\
\hline New surgery owing to complications & $6(10.9)$ & $9(16.4)$ & $15(13.6)$ & 0.40 \\
\hline Permanent stoma & $8(14.5)$ & 7 (12.7) & $15(13.6)$ & 0.78 \\
\hline 6 months chemotherapy regime & $18(32.7)$ & $14(25.5)$ & $32(29.1)$ & 0.40 \\
\hline
\end{tabular}

${ }^{*}$ Patients $<75$ years were included in the survey. $p$ Values calculated with $\chi$ square, $t$ test and Fisher's exact test when appropriate. GP, general practitioner.

Sensitivity analyses

The single factor with the greatest impact on overall societal costs was length of sick-leave, followed by cost of follow-up tests and cost of hospital travels. Variances in costs related to GP office travels and follow-up appointments had a minor impact on overall cost in a follow-up programme (figure 4).

Time to cancer diagnoses

A total of 48 SCEs occurred; the mean time until diagnosis of an SCE was 45 days in the surgeon group and 35 days in the GP group ( $p=0.46)$. Of the patients with SCE, 14 had cancer recurrence and $7(50 \%)$ were offered metastases surgery. Five patients died (all deaths caused by disseminated colon cancer) during the follow-up period (GP 1 vs surgeon 4).

\section{DISCUSSION}

Summary of findings

A representative population of patients surgically treated for colon cancer participated in this trial, with an 
Table 4 Health-related quality of life (ERTOC QLQ-C30, European Organisation for Research and Treatment of Cancer QoL Questionnaire; and EQ-5D, EuroQol-5D) outcome variables and estimated differences

\begin{tabular}{|c|c|c|c|c|c|}
\hline \multirow[b]{2}{*}{ Outcome variable } & \multicolumn{3}{|l|}{ Mean (SD) } & \multirow[b]{2}{*}{ Estimated mean difference $(95 \% \mathrm{Cl})$} & \multirow[b]{2}{*}{ p Value* } \\
\hline & Baseline & 12 months & 24 months & & \\
\hline \multicolumn{6}{|l|}{ Global health status } \\
\hline Surgeon & 70.7 (22.5) & $75.9(19.2)$ & $85.0(16.8)$ & & \\
\hline GP & $70.4(20.8)$ & $81.3(17.0)$ & 86.5 (16.2) & $-2.23(-5.7$ to 1.2$)$ & 0.20 \\
\hline \multicolumn{6}{|l|}{ Physical functioning } \\
\hline Surgeon & $80.5(23.6)$ & $88.8(15.0)$ & $88.0(17.0)$ & & \\
\hline GP & 74.5 (24.9) & $90.6(16.6)$ & $93.3(16.0)$ & $-2.4(-5.7$ to 0.8$)$ & 0.14 \\
\hline \multicolumn{6}{|l|}{ Role functioning } \\
\hline Surgeon & $62.5(37.3)$ & $83.8(26.5)$ & $90.3(18.6)$ & & \\
\hline GP & $62.7(37.5)$ & $91.6(22.1)$ & $93.7(20.7)$ & $-5.1(-9.7$ to $(-0.5))$ & 0.02 \\
\hline \multicolumn{6}{|l|}{ Emotional functioning } \\
\hline Surgeon & $87.4(18.1)$ & $87.7(16.1)$ & $87.7(16.9)$ & & \\
\hline GP & 85.8 (23.2) & $91.9(15.8)$ & $94.4(17.3)$ & $-3.7(-6.8$ to $(-0.6))$ & 0.01 \\
\hline \multicolumn{6}{|l|}{ Cognitive functioning } \\
\hline Surgeon & $87.0(20.6)$ & $86.5(22.8)$ & $90.3(15.0)$ & & \\
\hline GP & $72.4(31.8)$ & $91.1(17.0)$ & $93.0(21.3)$ & $-1.7(-5.0$ to 1.4$)$ & 0.27 \\
\hline \multicolumn{6}{|l|}{ Social functioning } \\
\hline Surgeon & $70.7(30.5)$ & $87.0(23.8)$ & $90.4(15.6)$ & & \\
\hline GP & $72.4(31.8)$ & $91.6(17.3)$ & $93.0(21.3)$ & $-4.2(-8.4$ to $(-0.009))$ & 0.04 \\
\hline \multicolumn{6}{|l|}{ Fatigue } \\
\hline Surgeon & $32.3(26.1)$ & $19.2(17.1)$ & $14.6(23.4)$ & & \\
\hline GP & $36.9(28.0)$ & $22.2(19.9)$ & $18.3(20.8)$ & $0.24(-3.7$ to 4.2$)$ & 0.9 \\
\hline \multicolumn{6}{|l|}{ Nausea and vomiting } \\
\hline Surgeon & $6.0(12.4)$ & $2.8(8.5)$ & $0.9(3.9)$ & & \\
\hline GP & $6.5(14.1)$ & $3.5(9.9)$ & $4.3(10.3)$ & $-0.8(-2.8$ to 1.2$)$ & 0.4 \\
\hline \multicolumn{6}{|l|}{ Pain } \\
\hline Surgeon & $22.3(26.6)$ & $11.1(21.9)$ & $9.6(16.9)$ & & \\
\hline GP & $19.1(28.2)$ & $9.3(14.0)$ & $2.8(14.7)$ & 4.5 (0.8 to 8.2$)$ & 0.01 \\
\hline \multicolumn{6}{|l|}{ Dyspnoea } \\
\hline Surgeon & $18.1(26.3)$ & $14.2(20.2)$ & $10.5(19.4)$ & & \\
\hline GP & $24.0(32.7)$ & $12.1(23.3)$ & $7.2(21.2)$ & $3.0(-1.2$ to 7.2$)$ & 0.1 \\
\hline \multicolumn{6}{|l|}{ Insomnia } \\
\hline Surgeon & $22.9(25.4)$ & $18.5(25.7)$ & $17.5(25.7)$ & & \\
\hline GP & $28.6(34.5)$ & $14.7(23.4)$ & $23.6(25.0)$ & $2.9(-1.7$ to 7.5$)$ & 0.2 \\
\hline \multicolumn{6}{|l|}{ Appetite loss } \\
\hline Surgeon & $15.5(23.1)$ & $3.7(10.6)$ & $1.7(7.6)$ & & \\
\hline GP & $20.9(31.7)$ & $1.9(7.9)$ & $4.1(11.2)$ & $0.8(-2.9$ to 3.9$)$ & 0.6 \\
\hline \multicolumn{6}{|l|}{ Constipation } \\
\hline Surgeon & $27.4(32.0)$ & $21.2(29.9)$ & $10.5(19.4)$ & & \\
\hline GP & $18.6(33.5)$ & $7.8(16.5)$ & $15.2(19.6)$ & $5.1(0.8$ to 9.4$)$ & 0.01 \\
\hline \multicolumn{6}{|l|}{ Diarrhoea } \\
\hline Surgeon & $24.4(29.6)$ & $21.2(25.3)$ & $24.5(24.4)$ & & \\
\hline GP & $31.0(33.6)$ & $22.5(26.8)$ & $23.6(28.6)$ & $-1.0(-5.7$ to 3.5$)$ & 0.6 \\
\hline \multicolumn{6}{|l|}{ Financial difficulties } \\
\hline Surgeon & $9.8(26.2)$ & $9.2(20.4)$ & $7.0(21.0)$ & & \\
\hline GP & $6.9(21.2)$ & $1.9(7.9)$ & $4.1(11.2)$ & $2.7(-0.4$ to 5.8$)$ & 0.08 \\
\hline \multicolumn{6}{|l|}{ EQ-5D Index score } \\
\hline Surgeon & $0.83(0.16)$ & $0.85(0.20)$ & $0.90(0.14)$ & & \\
\hline GP & $0.79(0.22)$ & $0.87(0.18)$ & $0.89(0.13)$ & $-0.10(-0.039$ to 0.018$)$ & 0.48 \\
\hline EQ-5D VAS score & & & & & \\
\hline Surgeon & $72.2(18.9)$ & $78.2(16.2)$ & $82.4(16.6)$ & & \\
\hline GP & $67.4(17.4)$ & $79.0(14.6)$ & $83.5(14.8)$ & $-1.10(-3.9$ to 1.7$)$ & 0.44 \\
\hline
\end{tabular}


(a)

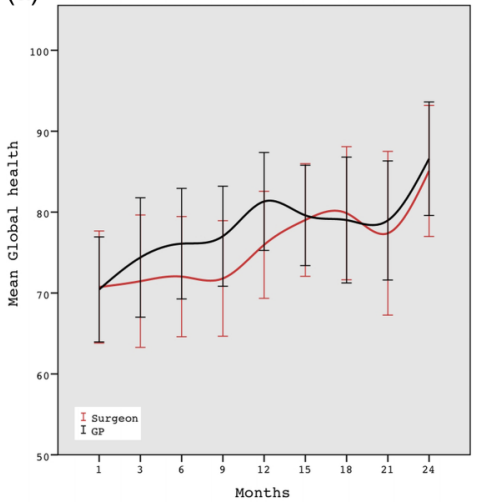

(b)

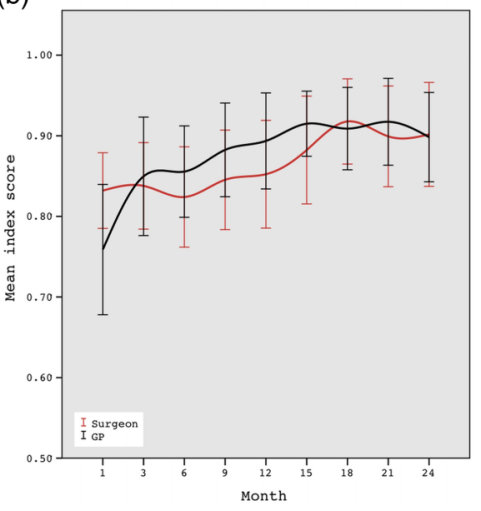

(c)

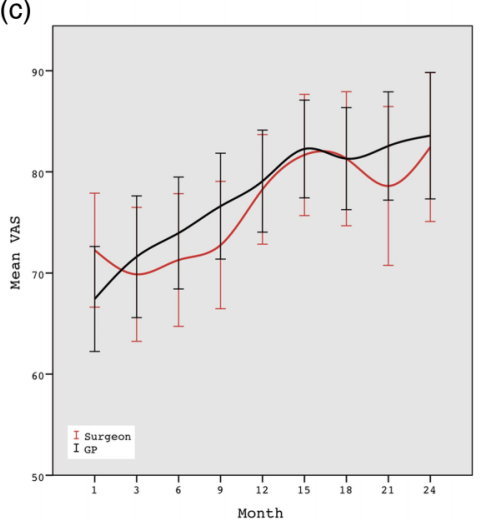

Figure 2 A-C. Health-related quality of life 1-24 postoperative months. European Organisation for Research and Treatment of Cancer QoL Questionnaire (EORTC QLQ C-30) Global Health, EuroQol-5D (EQ-5D) index score and EQ-5D visual analogue scale.

expected normal variance of demographic factors and colon cancer severity. We have shown that a decentralised colon cancer follow-up programme will not impair QoL; on the contrary, we observed a significant improvement in the following QoL subscales: role functioning, emotional functioning and pain. This is the first trial evaluating the economical implications of a GP organised follow-up programme after curative resection for colon cancer. Despite a higher frequency of healthcare contacts in primary care, a decentralised GP organised follow-up programme was associated with total cost savings owing to the decreased costs of primary care consultations and less hospital travels. Importantly, our result shows that GP follow-up was not associated with increased time to diagnosis of SCE, and thus cancer recurrence ( 35 vs 45 days, $\mathrm{p}=0.46$ ), and the frequency of an SCE was similar in both groups.

\section{Comparison with existing literature and ongoing trials}

Although intensive follow-up is associated with improved survival, there are still international controversies on how to best organise follow-up of colon cancer patients. These controversies are mirrored in the wide variation of national follow-up guidelines. ${ }^{4-7}$ Two systematic reviews, comparing follow-up trials, have been published. ${ }^{2}{ }^{3}$ Owing to the variation in the follow-up programmes included in these reviews, it is not possible to infer the best combination of consultations, blood tests, colonoscopy, radiological investigations and level of care to maximise the outcomes. ${ }^{2}$ Large randomised trials are under way (COLOFOL, GILDA and FACS), but the results are most likely years away. ${ }^{9-11}$ Few published surveys have evaluated the effect of a GP organised follow-up programme. Two surveys have reported on QoL in a primary care-based follow-up programme, and a single cost-effectiveness analysis of intensified hospitalbased follow-up was published in 2004. ${ }^{30-32}$ Surveys have assessed the costs of follow-up in a Norwegian setting. In a retrospective survey, 314 patients were assessed with regard to the cost, compliance and success rate of curative surgery. It was concluded that the cost of one successful curative surgery was US\$25 289, and that further implementation of such a programme should be debated. ${ }^{33}$ The harms and unintended effects of a follow-up programme are poorly explored. The rate of false positive tests in a follow-up programme is especially unknown. Current surveillance is often based on serial CEA measurements; this biomarker has several pitfalls and shortcomings. In a recent survey, it was shown that the diagnostic accuracy of the serial measurement of CEA is low, and is impacted by the cut-off value. ${ }^{34}$ These aspects are of high importance when designing a follow-up programme, as a false-positive test probably has a negative impact on the patient's QoL. Finally, there exists a considerable variance in follow-up strategies, internationally and at a national level. ${ }^{35}$ This makes the comparison of outcomes between different follow-up strategies challenging.

For other cancer conditions, more cost-effective ways of organising follow-up are extensively described and evaluated. For breast cancer patients, nurse lead telephone and GP organised follow-up are cost-effective ${ }^{36-38}$ with no increase in the frequency of SCE. ${ }^{39}$ Nevertheless, the quality of primary care cancer management is still debated. ${ }^{40-42}$

\section{Strengths and limitations}

Our trial has several strengths. First, this is the first randomised trial addressing the economical implications and time to recurrent cancer diagnoses in a GP-organised colon cancer follow-up programme. We have shown that GP-organised follow-up, even with the increased frequency of healthcare contacts, was associated with cost savings and no decline in QoL. Second, poor guideline compliance has been shown to represent a problem in cancer follow-up programmes. ${ }^{43}$ However, tools to support decision-making in cancer are in progress. In this study, a decision support pamphlet was part of the intervention, and the patient and the GP organising the follow-up received a decision support tool. We believe that this 
Table 5 Resource use in a colon cancer follow-up programme

\begin{tabular}{|c|c|c|c|c|c|c|c|c|c|}
\hline \multirow[b]{2}{*}{ Cost variable } & \multicolumn{2}{|c|}{ Surgeon $n=55$} & \multirow[b]{2}{*}{ cost/cycle } & \multicolumn{2}{|c|}{ GP $n=55$} & \multirow[b]{2}{*}{ cost/cycle } & \multicolumn{2}{|c|}{ Total $n=110$} & \multirow[b]{2}{*}{ cost/cycle } \\
\hline & $\bar{n}$ & n/cycle & & $\mathbf{n}$ & n/cycle & & $\mathbf{n}$ & n/cycle & \\
\hline Follow-up months & 903 & & & 897 & & & 1800 & & \\
\hline Car & 189 & 0.62 & * & 113 & 0.37 & * & 302 & 0.50 & * \\
\hline Taxi & 37 & 0.12 & & 22 & 0.07 & & 59 & 0.09 & \\
\hline Bus & 96 & 0.31 & & 33 & 0.11 & & 129 & 0.21 & \\
\hline $\begin{array}{l}\text { Extra travel owing } \\
\text { to poor logistics }\end{array}$ & 104 & 0.34 & & 52 & 0.17 & & 156 & 0.26 & \\
\hline Travel assistant & 59 & 0.19 & & 10 & 0.03 & & 69 & 0.11 & \\
\hline Hotel & 7 & 0.02 & $1.7(11)$ & 8 & 0.02 & $2.0(12)$ & 15 & 0.02 & $1.8(11.6)$ \\
\hline Total & $528^{*}$ & 1.75 & & $250^{*}$ & 0.83 & & $778^{*}$ & 1.29 & \\
\hline Taxi & 7 & 0.02 & & 14 & 0.05 & & 21 & 0.03 & \\
\hline Bus & 17 & 0.06 & & 35 & 0.12 & & 52 & 0.08 & \\
\hline Travel assistant & 0 & 0 & & 15 & 0.05 & & 15 & 0.02 & \\
\hline Total & 179 & 0.59 & & 381 & 1.27 & & 560 & 0.93 & \\
\hline Mean cost $£(S D)$ & & & $4.1(7.9)$ & & & $9.0(9.1, p<0.001)$ & & & $6.6(8.9)$ \\
\hline \multicolumn{10}{|l|}{$\begin{array}{l}\text { Out-of-pocket } \\
\text { expenses }\end{array}$} \\
\hline Mean cost $£(S D)$ & & & $2.7(7.7)$ & & & $4.3(15.0, p=0.10)$ & & & $3.5(11.9)$ \\
\hline \multicolumn{10}{|c|}{ Healthcare contacts } \\
\hline GP consultations & 156 & 0.52 & $9.6(17.8)$ & 329 & 1.10 & $20.6(19.9)$ & 485 & 0.80 & $15.1(19.6)$ \\
\hline $\begin{array}{l}\text { GP phone } \\
\text { consultation }\end{array}$ & 61 & 0.20 & $1.0(3.9)$ & 94 & 0.31 & $1.7(4.3)$ & 155 & 0.25 & $1.4(4.1)$ \\
\hline \multicolumn{10}{|l|}{$\begin{array}{l}\text { NGICG follow-up } \\
\text { tests }\end{array}$} \\
\hline Blood samples & 203 & 0.67 & $3.3(5.1)$ & 300 & 1.0 & $5.1(6.8)$ & 503 & 0.83 & $4.2(6.0)$ \\
\hline Chest x-ray & 150 & 0.50 & $12.2(12.2)$ & 128 & 0.43 & $10.6(12.1)$ & 278 & 0.46 & $11.4(12.2)$ \\
\hline CEUS & 110 & 0.37 & $56.2(74.0)$ & 99 & 0.33 & $51(72.5)$ & 209 & 0.34 & $53.8(73.2)$ \\
\hline Colonoscopy & 50 & 0.17 & $49.2(110.3)$ & 65 & 0.22 & $65.1(122)$ & 115 & 0.19 & $57.1(116.7)$ \\
\hline Total & 513 & 1.70 & & 592 & 1.97 & & 1105 & 1.84 & \\
\hline Mean cost $£(S D)$ & & & $121.1(152.8)$ & & & $132.2(166.7, p=0.39)$ & & & $126.6(159.8)$ \\
\hline \multicolumn{10}{|l|}{ Work loss } \\
\hline $\begin{array}{l}\text { Patients in paid } \\
\text { work }(n)\end{array}$ & 17 & & & 12 & & & 29 & & \\
\hline $\begin{array}{l}\text { Days off work } \\
\text { mean (SD) }\end{array}$ & $\begin{array}{l}215 \\
(168)\end{array}$ & & & $\begin{array}{l}198 \\
(190 \\
p=0.79)\end{array}$ & & & $\begin{array}{l}208 \\
(219)\end{array}$ & & \\
\hline $\begin{array}{l}\text { Mean cost } £(S D) \ddagger \\
\text { Serious clinical } \\
\text { events }\end{array}$ & & & $2440(1906)$ & & & $1884(2092, p=0.45)$ & & & $2086(2014)$ \\
\hline Number of events & 22 & & & 26 & & & 48 & & \\
\hline Mean cost $£(S D) \S$ & & & $261.6(157.7)$ & & & $\begin{array}{l}573.1 \\
(838.9, p=0.14\}\end{array}$ & & & $444.0(662.4)$ \\
\hline
\end{tabular}




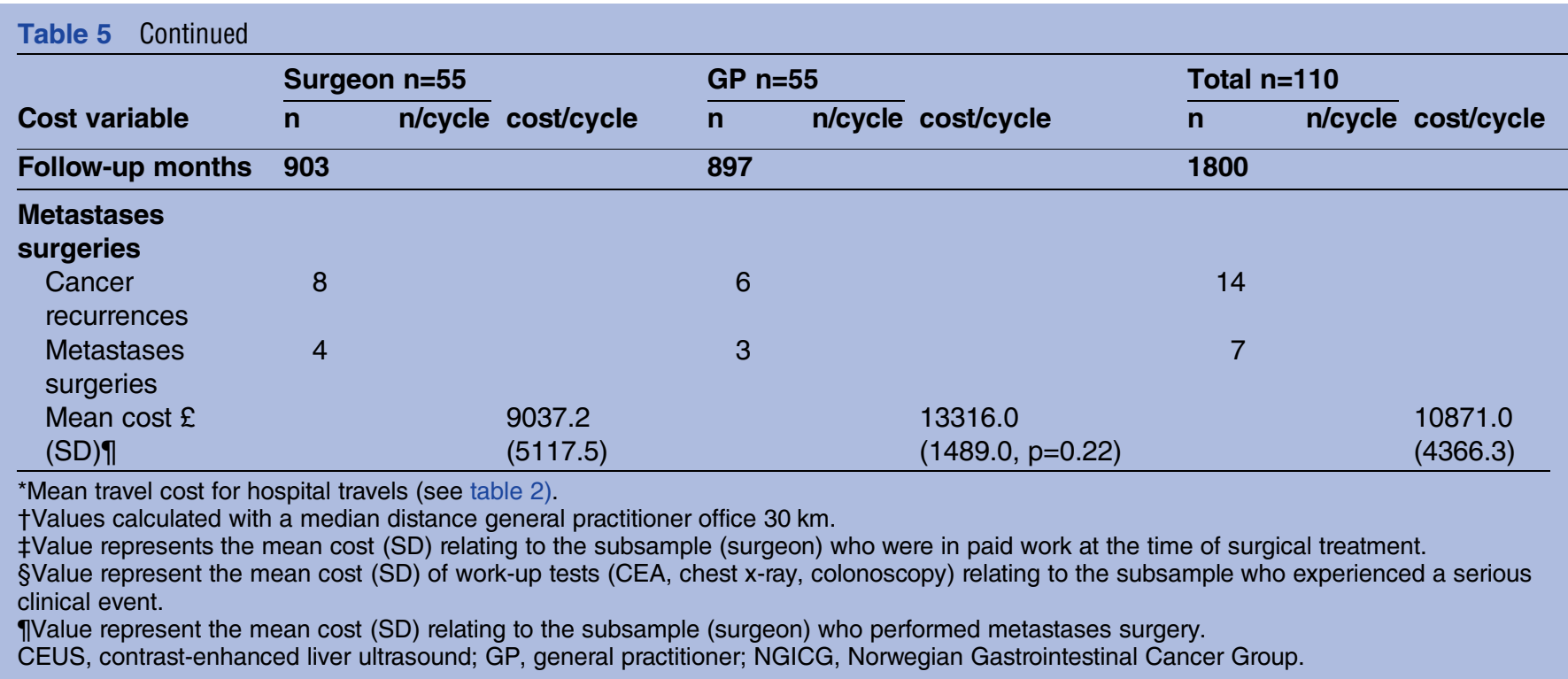

decision support tool contributed to a high follow-up guideline adherence (table 6, GP 592 tests vs surgeon 513 tests). Third, we have shown that the rate of SCE and time to diagnosis of cancer recurrence are comparable between groups. In our opinion, this is an indicator of adequate quality in a GP organised follow-up programme.

There exist limitations. First, it might be argued that we were missing important information by choosing another endpoint than survival. However, this trial was designed primarily to evaluate whether general practice follow-up results in effects on patient-specific QoL and costeffectiveness. We acknowledge that this choice of endpoint

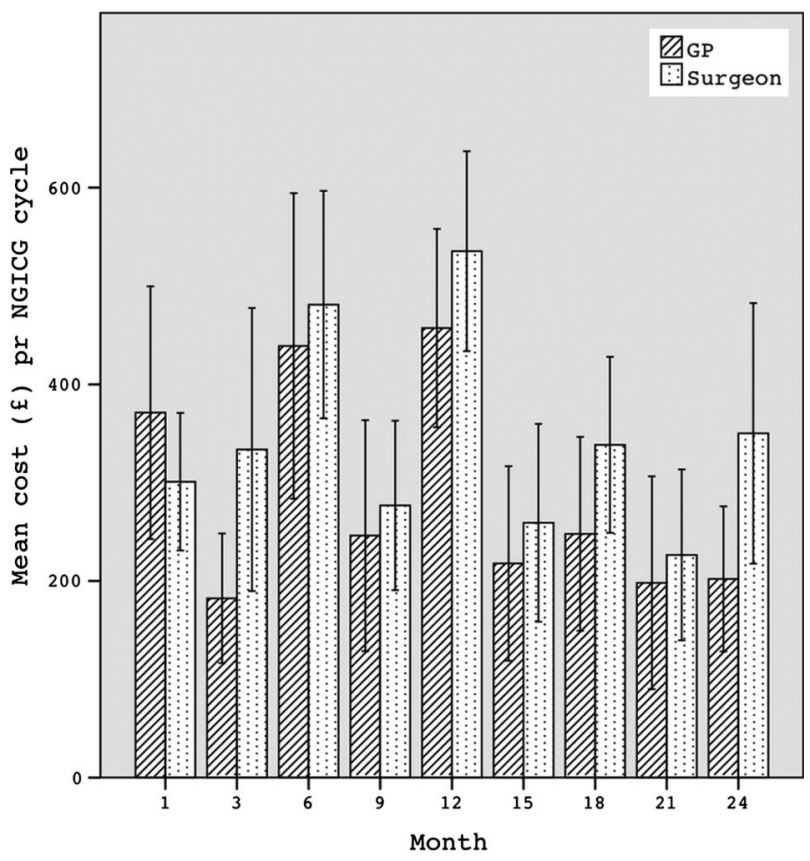

Figure 3 Health care cost of follow-up per 3 month follow-up cycle. might impact the observed frequency of SCEs and time to cancer diagnoses, as a higher number of SCE and cancer recurrences would have occurred with a longer follow-up time. Similarly, societal costs will be impacted by a longer follow-up time. However, when healthcare cost of follow-up is analysed separately (table 5, figure 3), cost spendings are significantly lower in the GP group compared to the surgeon group. Second, generalisability and cost transferability across jurisdictions might be challenging, as elements of cost data may vary from place to place. ${ }^{44}$ It might be argued that this is a single country trial with limited generalisability. However, we do not think this is the case. Comparable follow-up trials have been performed in countries like the USA, Canada, the UK, Australia and the Netherlands. ${ }^{30} 383945$ These surveys are commonly cited and thus accepted as generalisable. In Norway, the GP has a traditional gatekeeper function and plays a central role managing resource usage in secondary care. Similarly, many European countries have a healthcare organisation where the GP plays a central role as gatekeeper to the access of secondary healthcare service. In our trial, guidelines for dealing with aspects of generalisability and transferability were applied, and variations in unit costs were included in the sensitivity analyses (see figure 3). ${ }^{44}$

Finally, the trial was stopped after 1884 follow-up months owing to no significant effect of the intervention on global health score and implementation of a new national follow-up programme. This is a limitation, as it will impact the interpretation of cancer recurrence. However, it would have been unethical to spend large resources over years to complete an intervention with a $4 \%$ probability of showing a significant impact on global health score.

Implication for patients, decision makers and clinicians Colon cancer in numbers is the third largest cancer type worldwide and a considerable number of patients are 


\begin{tabular}{|c|c|c|c|c|}
\hline Cost variable (mean, $£$ ) & Surgeon $\mathrm{n}=55$ & GP $n=55$ & Total $n=110$ & p Value \\
\hline Healthcare cost/follow-up cycle & 351 & 292 & 324 & 0.02 \\
\hline Bootstrapped $95 \% \mathrm{Cl}$ & 315 to 386 & 255 to 327 & 296 to 348 & \\
\hline Mean difference $£$ & \multicolumn{2}{|c|}{58} & & \\
\hline Healthcare cost/24-month follow-up & 3178 & 2651 & 2917 & 0.03 \\
\hline Bootstrapped $95 \% \mathrm{Cl}$ & 2833 to 3485 & 2228 to 3006 & 2660 to 3147 & \\
\hline Mean difference $(£)$ & \multicolumn{2}{|r|}{ 年 } & & \\
\hline Societal cost/follow-up cycle & 1098 & 914 & 1007 & $<0.001$ \\
\hline Bootstrapped $95 \% \mathrm{Cl}$ & 1062 to 1139 & 877 to 954 & 981 to 1034 & \\
\hline Mean difference $(£)$ & \multicolumn{2}{|l|}{ 100 } & & \\
\hline Societal cost/24-month follow-up & 9889 & 8233 & 9068 & $<0.001$ \\
\hline Bootstrapped 95\% Cl & \multirow{2}{*}{\multicolumn{2}{|c|}{95091010194}} & 8823 to 9320 & \\
\hline Mean difference $(£)$ & & & & \\
\hline
\end{tabular}

enrolled in a postsurgical surveillance programme, resulting in significant societal cost. However, as there is no evidence-based consensus on how to design costeffective follow-up programmes, the differences in tests, test frequency and level of care will have a high impact on societal cost spending. Therefore, the cost-driving elements in a colon cancer follow-up programme have to be critically evaluated.

From a societal perspective, this survey has important implications. It may be argued that there are limited benefits from having GPs organising the follow-up programmes, as the radiological examinations and the colonoscopy have to be performed inhospital anyway. However, we believe that the most important factors causing a less costly GP follow-up are better coordination of care: as shown in table 5, GP organised follow-up leads to fewer hospital travels. We believe this is mainly caused by improved coordination of care, for instance by performing multiple radiological tests at the same hospital visit. Interestingly, the GP group had fewer extra travels (GP 52 travels vs surgeon 102 travels) owing to poor logistics (table 5). Cost of GP consultation versus hospital consultation: the societal cost of GP consultations is lower compared to cost of hospital consultations, owing to a more costly hospital infrastructure. Complex and chronic conditions: patients surgically treated often have other chronic illnesses, and there is a trend towards higher involvement of primary care in treating these conditions as described in the chronic care model. ${ }^{\text {S }}$ Sick leave. although not statistically significant, patients in the GP group return to work 17 days (mean) earlier compared to patients in the surgeon group.

From a hospital perspective, a transfer of follow-up programmes to primary care has economical and organisational implications. GP organised follow-up may be an effective way of reducing the burden on busy hospital clinics.
Figure 4 Sensitivity analyses of cost-driving elements in surveillance. Societal cost per patient $(£)$ for a 24-month colon cancer follow-up. Most critical variable in terms of impact is listed at the top of the graph, and the rest ranked according to their impact thereafter. As unit cost from the UK, like cost for GP consultation and diagnostic testing, has been reported to be $30-40 \%$ higher than units cost applied in this trial, relevant cost elements were increased accordingly. Cost values for serious clinical events, metastases surgeries and sick leave were adjusted for baseline characteristics.

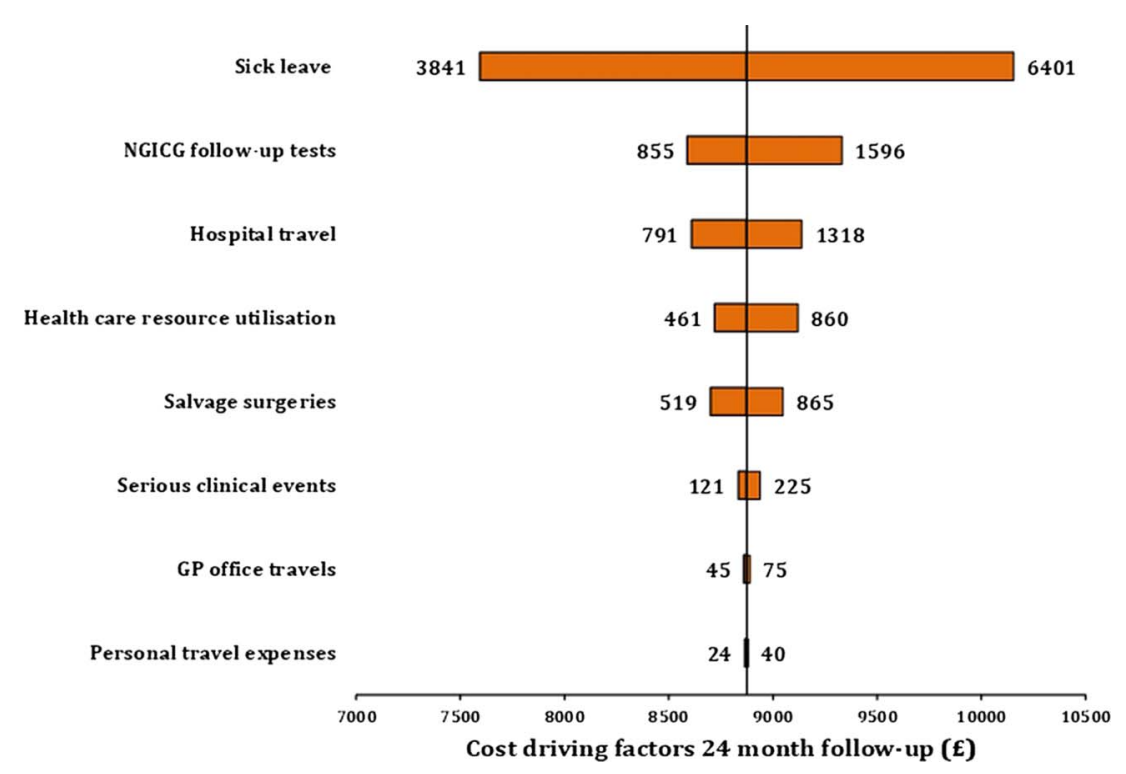




\section{CONCLUSION}

The present study suggests that a colon cancer follow-up can safely be performed by GPs, with no negative impact on QoL and at a lower cost. However, there exist limitations. Thirteen per cent $(n=14)$ of patients had colon cancer recurrence; this low recurrence rate is most likely caused by limited long-term follow-up as most recurrences occur within 3 years. Furthermore, the best combination of consultations, radiological test, blood samples and colonoscopy that optimises cancer survival is unknown. We therefore argue that the cost-driving elements of colon cancer surveillance should be critically evaluated, when implementing follow-up programmes, as cancer surveillance represents a huge financial burden for the society. Finally, little is known about the potential harms of follow-up, especially when it comes to the impact of false positive tests. Further research is needed to settle these controversies, and new methods of decision-analytic modelling in combination with emerging data from ongoing randomised trials must be applied. $^{46}$

\section{Author affiliations}

${ }^{1}$ Norwegian Center of Integrated Care and Telemedicine, University Hospital of North Norway, Tromsø, Norway

${ }^{2}$ Department of Gastrointestinal Surgery, University Hospital of North Norway, Tromsø, Norway

${ }^{3}$ Faculty of Health Sciences, Institute of Clinical Medicine, University of Tromsø, Tromsø, Norway

${ }^{4}$ Northern Norway Regional Health Authority Trust, Bodø, Norway

${ }^{5}$ Department of Surgery, Helgeland Hospital, Mo i Rana, Norway.

${ }^{6}$ Nordbyen Primary Care Office, Tromsø, Norway

${ }^{7}$ Department of Surgery, Nordland Hospital Trust, Bodø, Norway

Acknowledgements We thank Trine Hansen at the Norwegian Centre for Integrated Care and Telemedicine for administrative support and for maintaining the research database. We thank Professor Roar Johnsen, Department of Public Health and General Practice, Norwegian University of Science and Technology, for assistance in protocol writing and design of the trial. We thank Professor Lars Vatten, Department of Epidemiology, Norwegian University of Science and Technology, for assistance in prestudy sample size calculations. We thank Johnie Rose, MD, PhD, Department of Family Medicine and Community Health, Case Western Reserve University, for valuable comments on our research and manuscript. We thank Dr Caroline Sagatun (Surgical Department, Bodø Regional Hospital) and Dr Henriette Fagertun (Surgical Department, Harstad Hospital) for comments on the study protocol and identification of the potential trial participants. We thank Frank Hauboff (Surgical Outpatient Clinic, University Hospital of North Norway) for assistance in randomisation and identification of potential trial participants. We thank Berit Marianne Bjelkåsen, Norwegian University of Science and Technology, for assistance with the web-based randomisation service.

Contributors KMA and ROL conceived and designed the research idea, and were responsible for the overall administration and direction of the study, as well as the analysis and interpretation of the data. KMA and SOS designed the statistical analyses, while KMA did the statistical analyses. KMA did the economic analysis with assistance from JN, who contributed to the design, data analysis and interpretation of the findings. TN, RA and SD helped with patient recruitment and randomisation, and with the trial and interpretation of the findings. UR advised on the trial protocol, unit cost and reimbursement practice in primary care. BV advised on protocol writing and on the pretrial sample size calculations and manuscript revision. KMA wrote the first draft. All authors read and approved the final manuscript. KMA had full access to all the data in the study and had final responsibility for the decision to submit for publication.

Funding Northern Norway Health Authorities Research Fund.

Competing interests http://www.icmje.org/coi_disclosure.pdf None.

Patient consent Obtained.

Ethics approval IRB board University Hospital North Norway.

Provenance and peer review Not commissioned; externally peer reviewed.

Data sharing statement No additional data are available.

\section{REFERENCES}

1. Larsen IK. Cancer in Norway 2009. Oslo, Norway: Cancer Registry of Norway, 2011:1-169.

2. Jeffery M, Hickey BE, Hider PN. Follow-up strategies for patients treated for non-metastatic colorectal cancer. Cochrane Database Syst Rev 2007; doi:10.1002/14651858.CD002200.pub2

3. Tjandra JJ, Chan MKY. Follow-up after curative resection of colorectal cancer: a meta-analysis. Dis Colon Rectum 2007;50:1783-99.

4. Figueredo A, Rumble RB, Maroun J, et al. Follow-up of patients with curatively resected colorectal cancer: a practice guideline. $B M C$ Cancer 2003;3:26.

5. Cairns SR, Scholefield JH, Steele RJ, et al. Guidelines for colorectal cancer screening and surveillance in moderate and high risk groups (update from 2002). Gut 2010;59:666e690. doi:10.1136/gut.2009. 179804

6. Bülow S. Retningslinier for diagnostik og behandling af kolorektal cancer (Danish Guidelines). Danish Colorectal Cancer Group, 2009;4:1-176

7. Vonen B. Nasjonalt handlingsprogram med retningslinjer for diagnostikk, behandling og oppfølging av kreft i tynntarm og endetarm (Norwegian Guidelines). Norwegian Gastrointestinal Cancer Group NGICG, 2010:1-162.

8. Engstrom PF. NCCN clinical practice guidelines in oncology colon cancer V.2.2009. National Comprehensive Cancer network, 2009;2:1-71. www.ncen.org

9. Follow-up study of patients who have undergone surgery for stage I, stage II, or stage III colorectal cancer. clinicaltrials.gov. 2009:1-4.

10. Grossmann EM, Johnson FE, Virgo KS, et al. Follow-up of colorecta cancer patients after resection with curative intent-the GILDA trial. Surg Oncol 2004;13:119-24.

11. Wille-Jørgensen $P$, Laurberg $S$, Carriquiry $L$, et al. An interim analysis of recruitment to the COLOFOL trial. Colorect Dis 2009;11:756-8.

12. Wille-Jørgensen P, Balleby L. Follow-up in colorectal cancer: questions to be answered. Colorectal Dis 2011;13:959-60.

13. Bodenheimer T, Wagner EH, Grumbach K. Improving primary care for patients with chronic illness. JAMA 2002;288:1775-9.

14. National Cancer Survivor Initiative. Department of Health, Macmillan Cancer Support and NHS Improvement, London, UK, 2010;1-82.

15. Augestad KM, Vonen B, Aspevik R, et al. Should the surgeon or the general practitioner (GP) follow up patients after surgery for colon cancer? A randomized controlled trial protocol focusing on quality of life, cost-effectiveness and serious clinical events. BMC Health Serv Res 2008;8:137.

16. Campbell NC. Designing and evaluating complex interventions to improve health care. BMJ 2007;334:455-9.

17. Kawamoto K. Improving clinical practice using clinical decision support systems: a systematic review of trials to identify features critical to success. BMJ 2005;330:765-70.

18. Sprangers MA, Cull A, Bjordal K, et al. The European Organization for Research and Treatment of Cancer. Approach to quality of life assessment: guidelines for developing questionnaire modules. EORTC study group on quality of life. Qual Life Res 1993;2:287-95.

19. Dolan P. Modeling valuations for EuroQol health states. Med Care 1997;35:1095-108.

20. Dolan P. The time trade-off method: results from a general population study. Health Econ 2006;5:1-14.

21. Cambell M. Estimating sample sizes for binary, ordered categorical, and continuous outcomes in two group comparisons. BMJ 1995;311:1145-7.

22. King $M$. The interpretation of scores from the EORTC quality of life questionnaire QLQ-C30. Qual Life Res 2004:5:555-67. 
23. Drummond M. Guidelines for authors and peer reviewers of economic submissions to the BMJ. BMJ 1996;313:275-83.

24. Drummond M, Sculpher MJ, Torrance GW. Methods for the economic evaluation of health care programmes. New York: Oxford University Press Inc, 2005.

25. Desgagné A, Castilloux A, Angers $\mathrm{J}$, et al. The use of the bootstrap statistical method for the pharmacoeconomic cost analysis of skewed data. Pharmacoeconomics 1998;13:487-97.

26. Hill JC, Whitehurst $\mathrm{D}$, Lewis $\mathrm{M}$, et al. Comparison of stratified primary care management for low back pain with current best practice (STarT Back): a randomised controlled trial. Lancet 2011;378:1560-71.

27. Lachin JM. Futility interim monitoring with control of type I and II error probabilities using the interim Z-value or confidence limit. Clin Trials 2009;6:565-73.

28. Lachin JM. A review of methods for futility stopping based on conditional power. Stat Med 2005;24:2747-64.

29. Jitlal M, Khan I, Lee SM, et al. Stopping clinical trials early for futility: retrospective analysis of several randomised clinical studies. $\mathrm{Br} \mathrm{J}$ Cancer 2012;107:910-17.

30. Wattchow DA, Weller DP, Esterman A, et al. General practice vs surgical-based follow-up for patients with colon cancer: randomised controlled trial. Br J Cancer 2006;94:1116-21.

31. Renehan AG, O'Dwyer ST, Whynes DK. Cost effectiveness analysis of intensive versus conventional follow up after curative resection for colorectal cancer. BMJ 2004;328:1-5.

32. Gall CA, Weller D, Esterman A, et al. Patient satisfaction and health-related quality of life after treatment for colon cancer. Dis Colon Rectum 2007;50:801-9.

33. Körner H, Søreide K, Stokkeland PJ, et al. Systematic follow-up after curative surgery for colorectal cancer in Norway: a population-based audit of effectiveness, costs, and compliance. J Gastroint Surg 2005;9:320-8.

34. Körner H, Søreide K, Stokkeland PJ, et al. Diagnostic accuracy of serum-carcinoembryonic antigen in recurrent colorectal cancer: a receiver operating characteristic curve analysis. Ann Surg Oncol 2006;14:417-23.
35. Søreide K, Traeland JH, Stokkeland PJ, et al. Adherence to national guidelines for surveillance after curative resection of nonmetastatic colon and rectum cancer: a survey among Norwegian gastrointestinal surgeons. Colorectal Dis 2012;14 $320-4$.

36. Beaver $\mathrm{K}$, Hollingworth $\mathrm{W}, \mathrm{McD}$ Donald R, et al. Economic evaluation of a randomized clinical trial of hospital versustelephone follow-up after treatment for breast cancer. Br J Surg 2009;96:1406-15.

37. Grunfeld E. Follow-up of breast cancer in primary care vs specialist care: results of an economic evaluation. Br J Cancer 1999;79:1227-33.

38. Kimman ML, Dirksen CD, Voogd AC, et al. Economic evaluation of four follow-up strategies after curative treatment for breast cancer: results of an RCT. Eur J Cancer 2011;47:1175-85.

39. Grunfeld E. Randomized trial of long-term follow-up for early-stage breast cancer: a comparison of family physician versus specialist care. J Clin Oncol 2006;24:848-55.

40. Editorial. Cancer detection and primary care revisited. Lancet Oncol 2012;13:559.

41. Rubin G, Lyratzopoulos G, Abel G, et al. Cancer detection in primary care. Lancet Oncol 2012;13:e325-6.

42. Lyratzopoulos G, Neal R, Barbiere J, et al. Variation in number of general practitioner consultations before hospital referral for cancer: findings from the 2010 national cancer patient experience survey in England. Lancet Oncol 2012;13:353-65.

43. Cooper G, Kou T, Reynolds H. Receipt of guideline-recommended follow-up in older colorectal cancer survivors. Cancer 2008;113:2029-37.

44. Drummond $\mathrm{M}$, Barbieri $\mathrm{M}, \mathrm{Cook} \mathrm{J}$, et al. Transferability of economic evaluations across jurisdictions: ISPOR good research practices task force report. Value Health 2009;12:409-18.

45. Grunfeld E. Routine follow up of breast cancer in primary care: randomised trial. BMJ 1996;313:665-9.

46. Smith RA. Mathematical models and cost-effective screening strategies for colorectal cancer. CMAJ 2010;182:1283-4. 
